\title{
SPECIFIC EMG FINDINGS IN CASES OF NEUROGENIC BLADDER AND RECTAL DISTURBANCES
}

\author{
By Professor M. L. Allert ${ }^{1}$, F. Jelasic ${ }^{2}$ and H. Schneider ${ }^{3}$ \\ University Clinic, 665 Homburg (Saar), Germany
}

WITH electromyography we are able to trace the innervation of striated muscle. Thus, by the use of this method, we are also able to examine the striated sphincters of the bladder and of the anus. However, the electromyograph cannot measure the activity of the detrusor muscle of the bladder which is innervated by the autonomic nervous system (parasympathetic). The involuntary and voluntary nervous structures which innervate the bladder and anus are anatomically and functionally closely related; this is the reason why important information needed for judging the normal and pathological function of bladder and rectum can be achieved by tracing the bio-electrical activity of striated muscles of the sphincters and of the muscles of the pelvic floor.

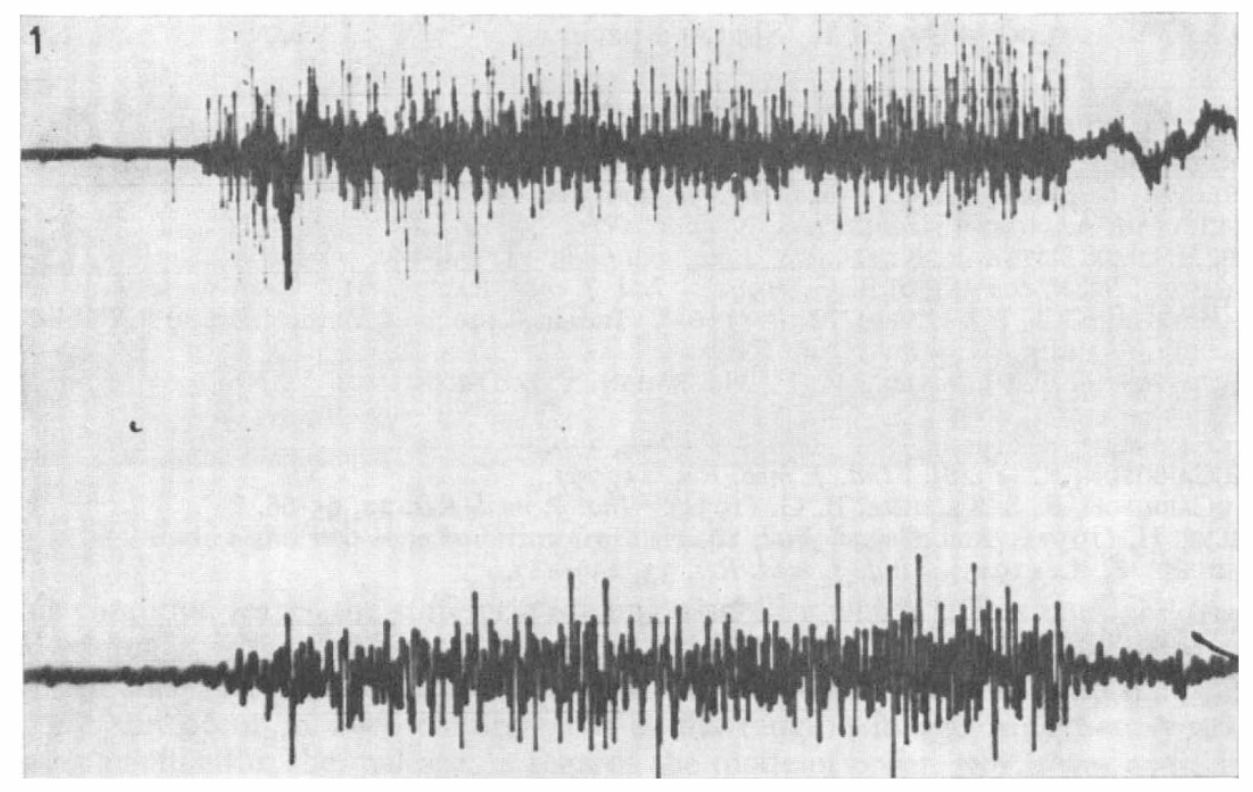

FIG. I

Interference patterns showing normal intensive activity during voluntary innervation. The above pattern shows a tracing from the Musculus sphincter ani externus (s.a.), and the lower tracing is from the Musculus sphincter vesicae externus (s.v.).

1 Present address: Department of Neurology and Psychiatry Bundeswehrzentralkrankenhaus, 54 Koblenz, Germany

2 Neuro-Surgical University Clinic, 665 Homburg (Saar).

3 University Neuro-Psychiatric Clinic, 665 Homburg (Saar). 
The examination technique is quite simple. Under rectal control of a finger, a needle electrode is inserted transperineally up to and underneath the lower pole of the prostate gland where one finds the Musculus sphincter vesicae externus or the Musculus transversus perinaei profundus. When using the method on a woman, it is recommended, for the sake of better orientation, to use a urethral catheter

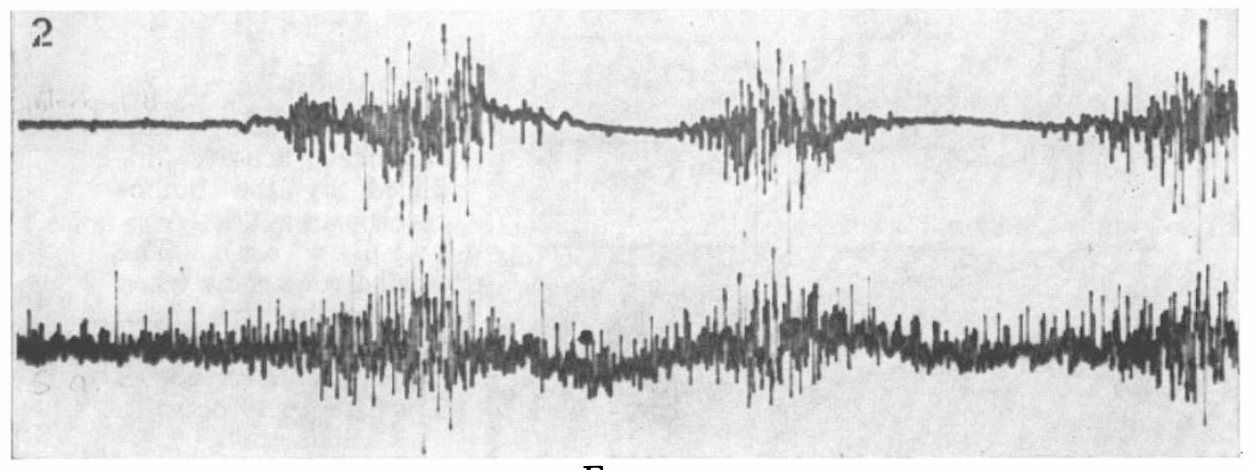

FIG. 2

The bottom tracing shows reflex activity from s.a. induced by coughs. The other tracing is a control taken from the Musculi intercostales.

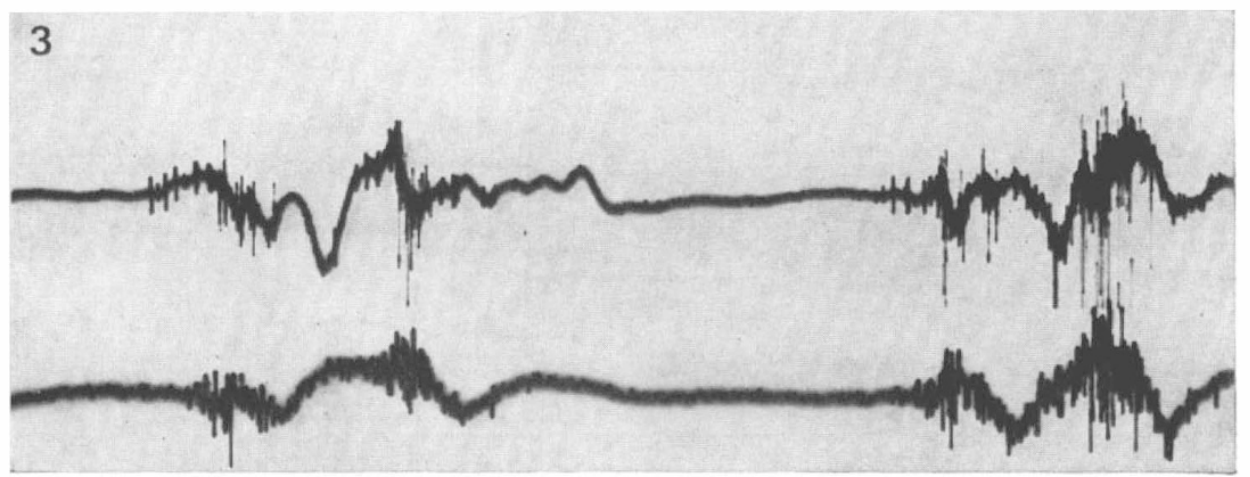

Fig. 3

The top tracing shows reflex activity in the s.a., and the bottom curve shows similar activity from the s.v. when rhythmic pressure is exerted upon the lower abdomen.

which can be removed when the electrode is in place. The registering electrode can also easily be inserted into the sphincter ani externus under digital control.

In principle, the following aspects of the electrical activity of striated sphincters are examined: at rest, active voluntary contraction and relaxation, and finally reflex contraction. In this way, it can be determined if a disturbance of innervation exists, to what extent the impulse transmission is impaired and if a lesion of the central or peripheral neuron is present.

In normal persons during voluntary contraction an intensive electrical activity in the sense of interference pattern appears (fig. I).

The same type of intensive electrical activity is seen in such reflex activities 


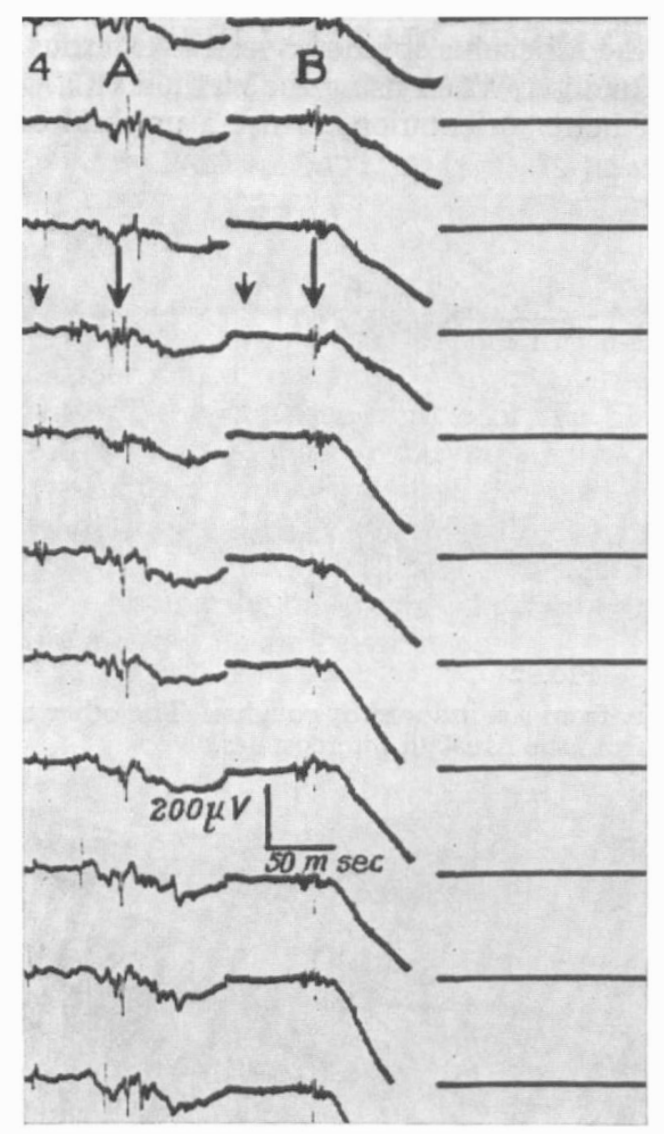

FIG. 4

The above tracings show electrical activity induced by the bulbocavernosus reflex $(\mathrm{A}=$ s.v.; $\mathrm{B}=$ s.a.). The small arrows show when the stimulus was given, the larger arrows show the stimulus response. The latency is between the arrows.

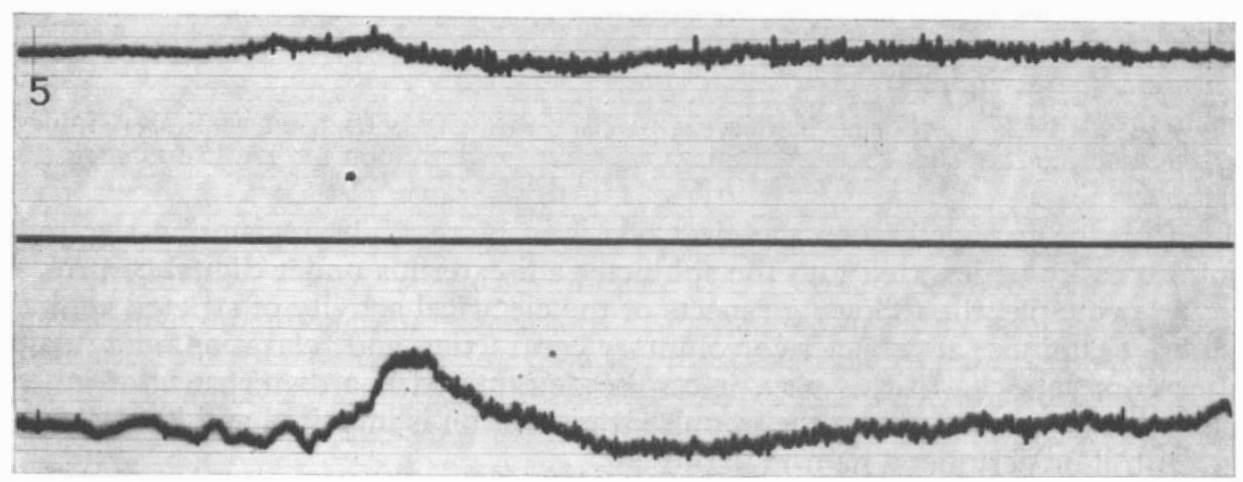

FIG. 5

Lesion of a peripheral neuron: Definite reduction of potentials and rarefied activity pattern (top tracing $=$ s.a., bottom tracing $=$ s.v.). 
6

FIG. 6

Lesion of a peripheral neuron: EMG tracing without potentials. The middle curve on the left shows fibrillations (s.a.).

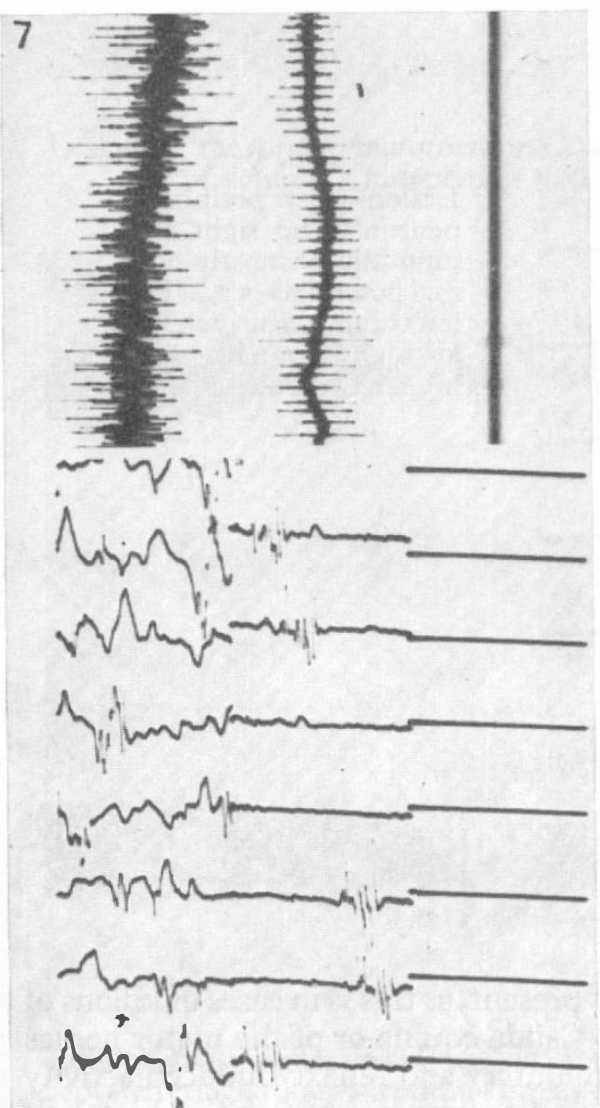

FIG. 7

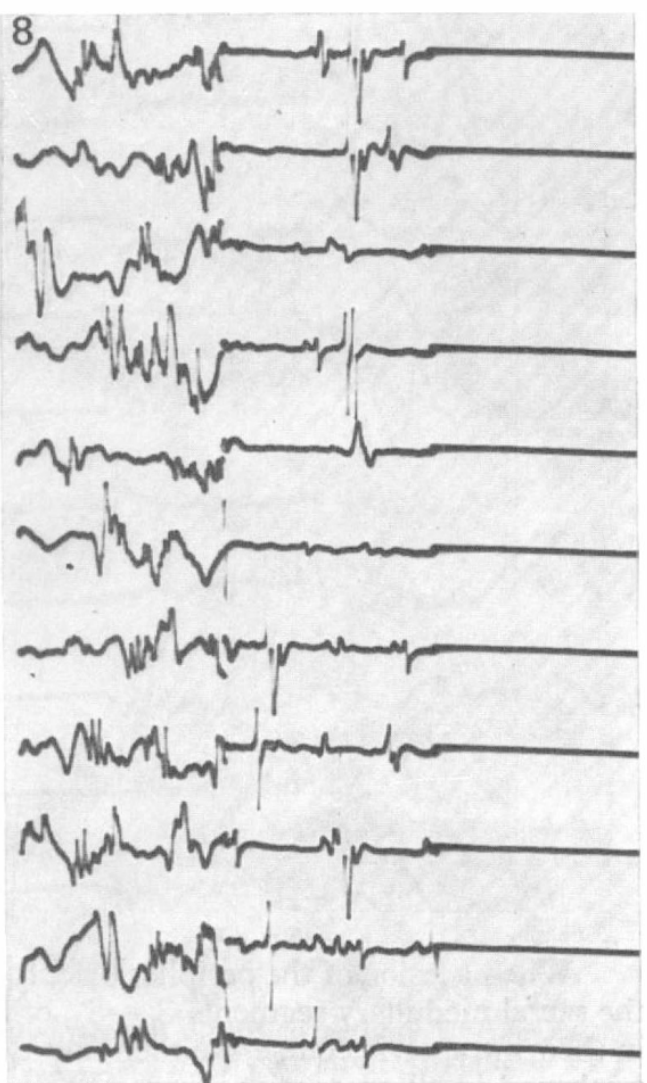

Fig. 8

Fig. 7. Lesion of a peripheral neuron: Polyphasic units and fasciculations (right column showing activity from the s.a. shows a more extensive lesion than that of the left column taken from the s.v.).

Fig. 8. Lesion of a peripheral neuron: The right column for the most part shows normal potentials (s.a.). The left column shows an increase in polyphasic units and a longer potential duration (s.v.). 
which the patients neither directly nor indirectly control. Examples of such reflex activities can be seen when a patient coughs (fig. 2), when rhythmic pressure is exerted upon the lower portion of the abdomen (fig. 3), or in a case of the bulbocavernosus reflex (fig. 4).

When disturbances of innervation are present, the electrical activity is more or less changed according to the extent of the lesion.

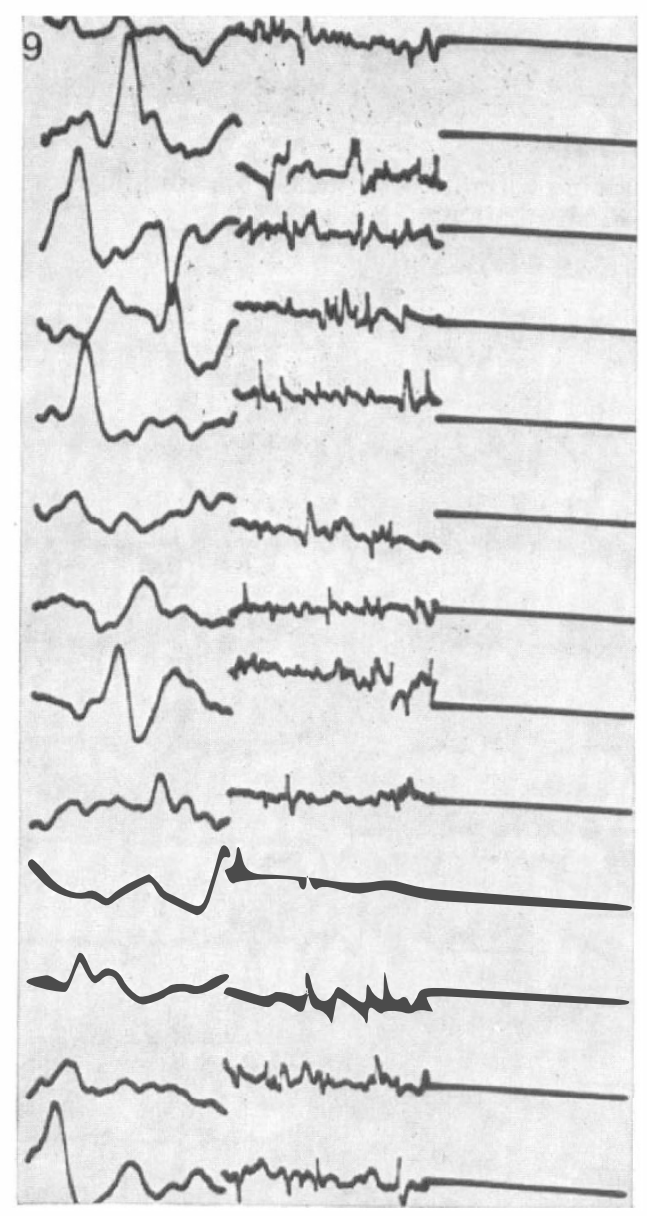

FIG. 9

Lesion of a peripheral neuron: The right column shows mostly normal potentials (s.a.). The left column clearly shows longer potentials with higher amplitudes (s.v.).

When a lesion of the peripheral neuron is present, as this is in cases of lesions of the sacral medullary segments $S_{2}-S_{4}$, of the Cauda equina or of the motor nerves in their intrapelvic course, we then find the voluntary and reflexly induced activity weakened in all graduations from merely a small reduction as shown in Figure 5, to the complete cessation of all activity as shown in Figure 6.

Moreover, the EMG in a majority of cases shows denervation potentials, fibrillations (fig. 6), an increase in polyphasic units (fig. 7) and an increased duration (fig. 8 and 9).

These last mentioned EMG changes are typical for lesions of the peripheral 
motor neuron. We can then in cases of lesions of the sacral spinal cord, of roots or of Nervi pudendi, the extent of the injury can be determined. In this way, a prognosis can more easily be given and often therapeutic possibilities are open.

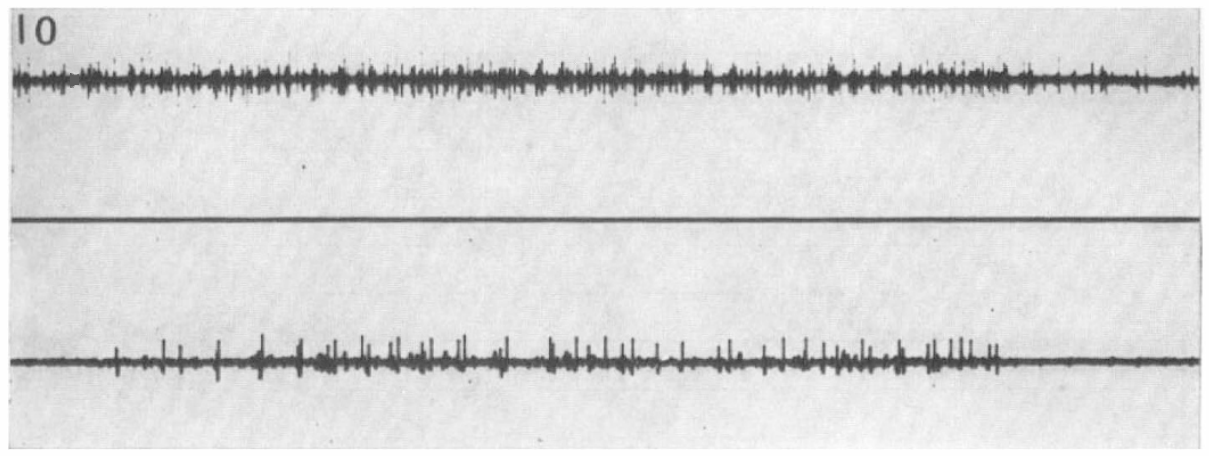

FIG. IO

Lesion of the upper motorneuron: Diminished rarefied activity pattern in the case of voluntary innervation (top tracing $=$ s.a.; lower tracing $=$ s.v.).

\section{1}
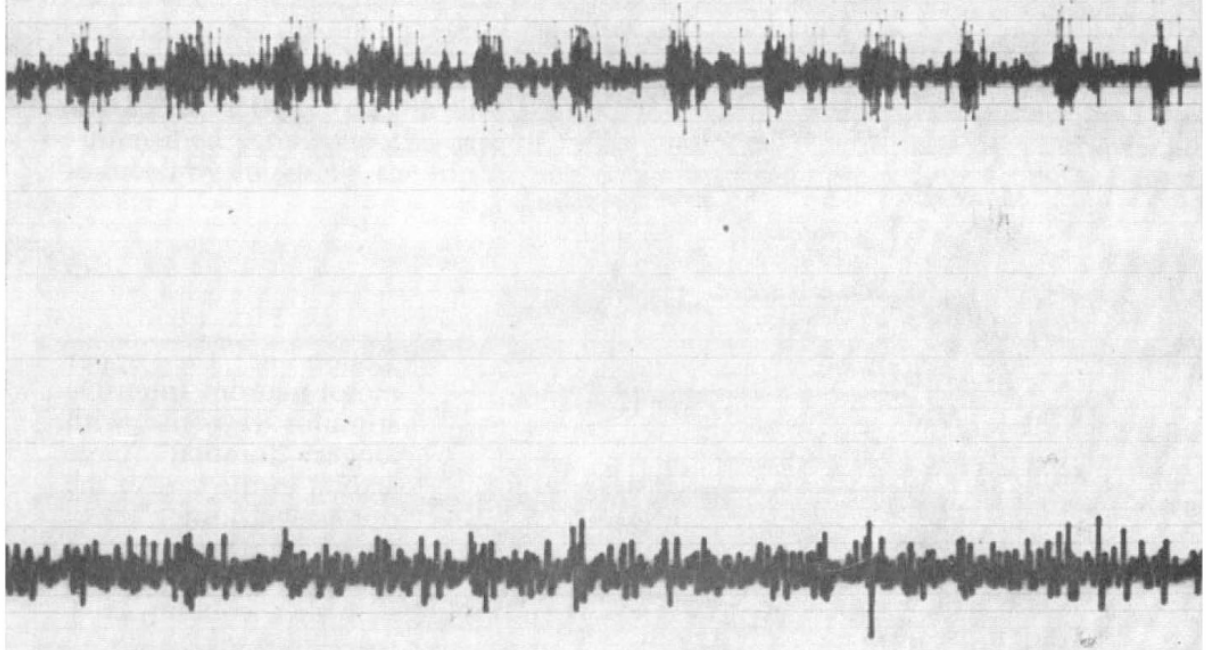

FIG. II

Lesion of the upper motor neuton: Reflexly induced electrical activity by means of repeated coughs shows a normal intensity of the interference pattern (top tracing $=$ s.a.; lower tracing $=$ s.v.). Compare with Figure 2.

In the case of a lesion of the upper motor neuron, that is in cases of lesions above the sacral segments, such typical potentials are not present. However, one does find characteristic changes which point in a direction of a upper motor neuron lesion. When a lesion of the upper motor neuron is present, the electrical 


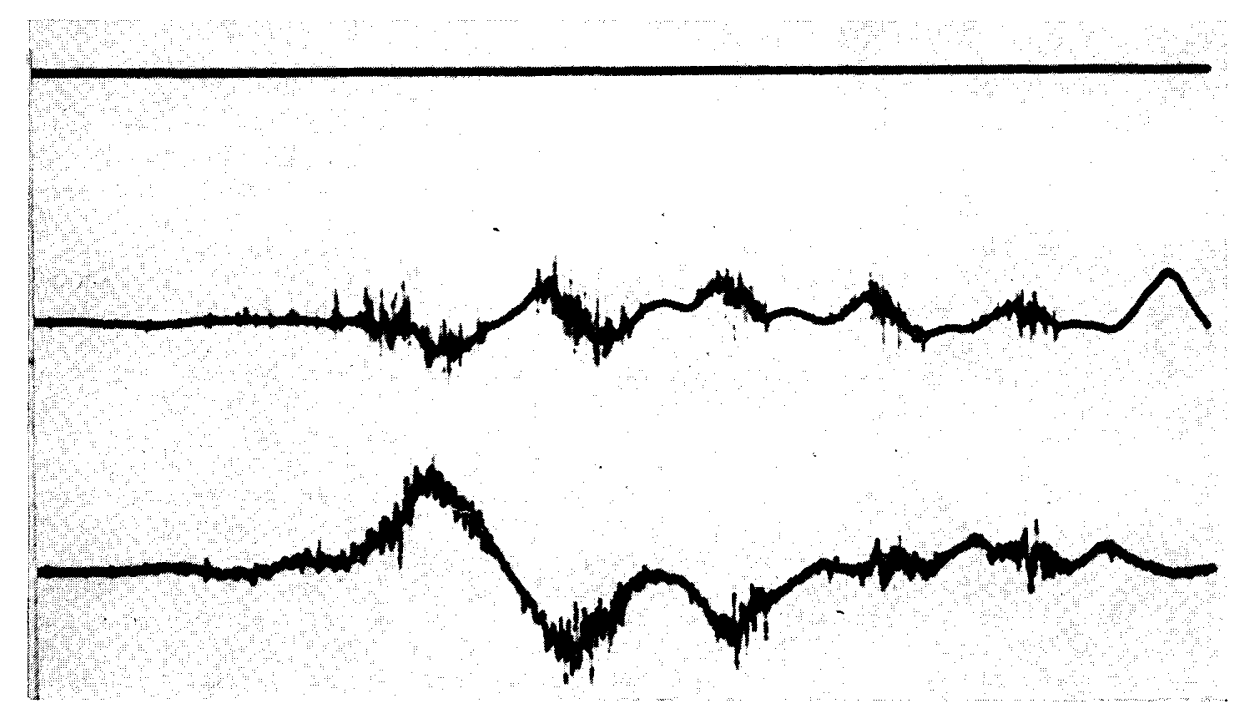

FIG. I2

Lesion of the upper motor neuron: Activity reflexly induced by rhythmic pressure on the abdomen. The tracings show very intensive interference patterns (top tracing = s.a.; lower tracing $=$ s.v.). Compare also with Figure 3 .

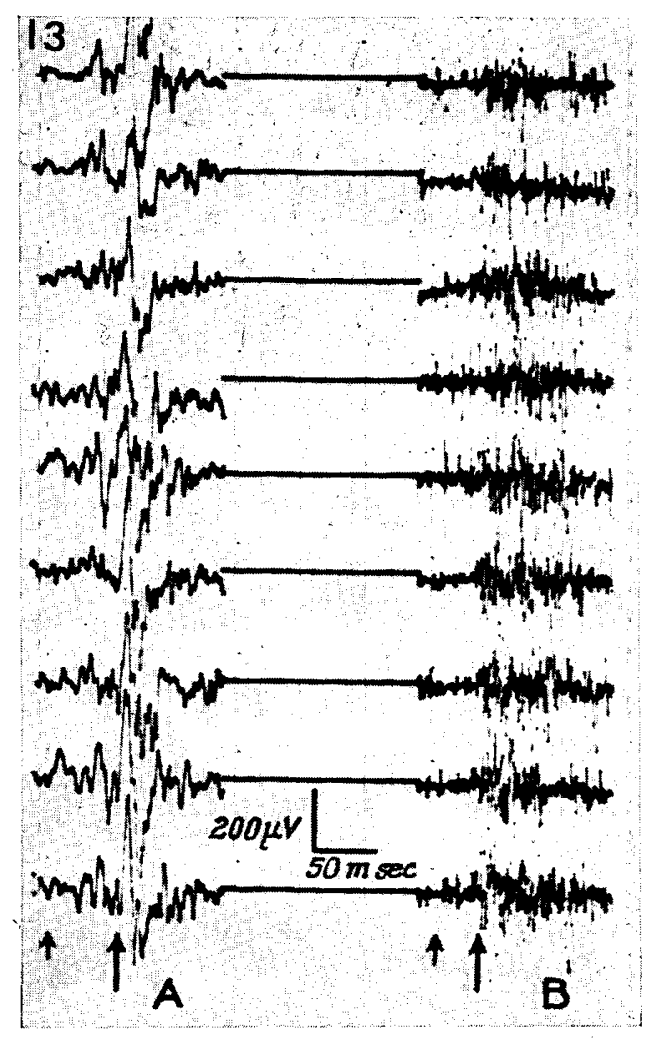

FIG. 13

Lesion of the upper motor neuron: Intensive stimulus response with longer duration (large arrow) in the case of the bulbocavernosus reflex (small arrow $=$ start of the stimulation) (column $\mathrm{B}=$ s.a.; column $\mathrm{A}=$ S.v.). 


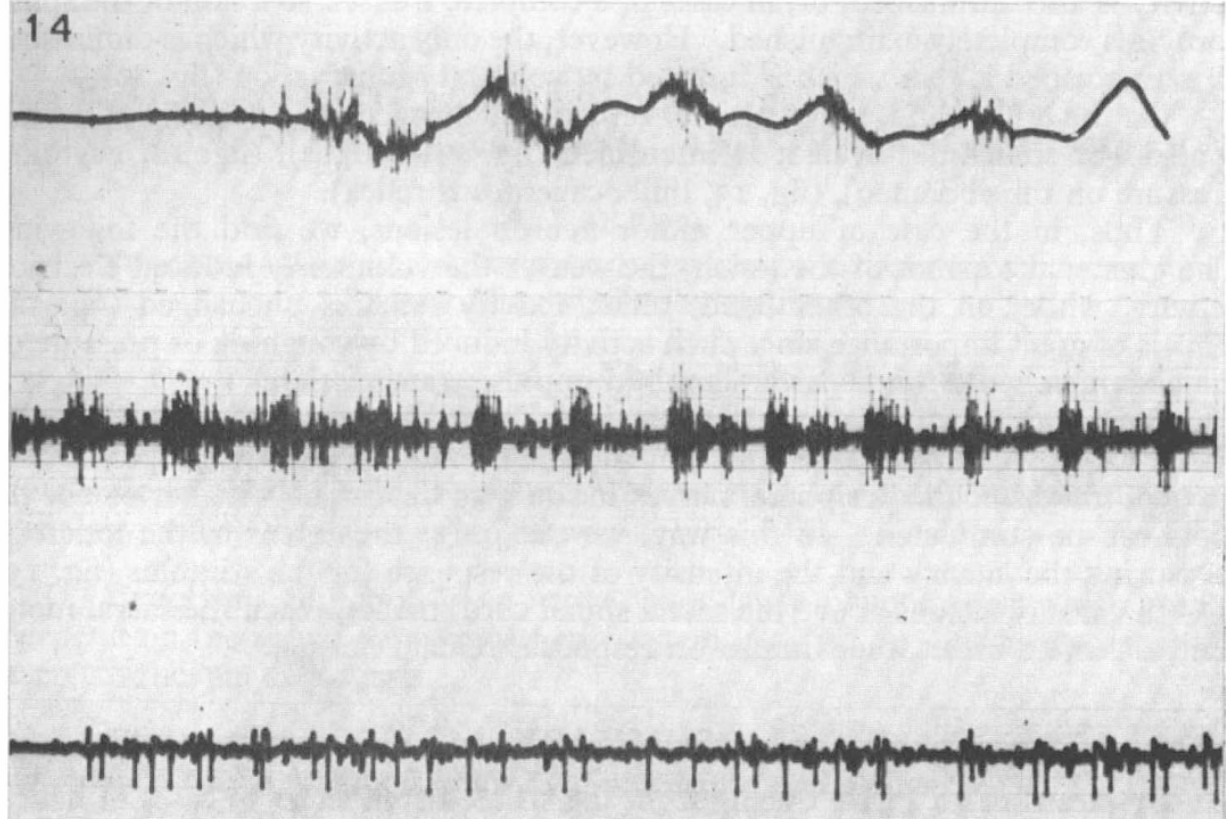

FIG. I4

Divergence of EMG findings in the case of a upper motor neuron lesion: Reduced bioelectrical activity (s.a.) in the case of voluntary innervation (lower tracing). Normal or intensified activity in the case of reflex induction (the middle curve shows activity induced by coughing, the top tracing activity induced by pressure on the abdomen).

\section{5}

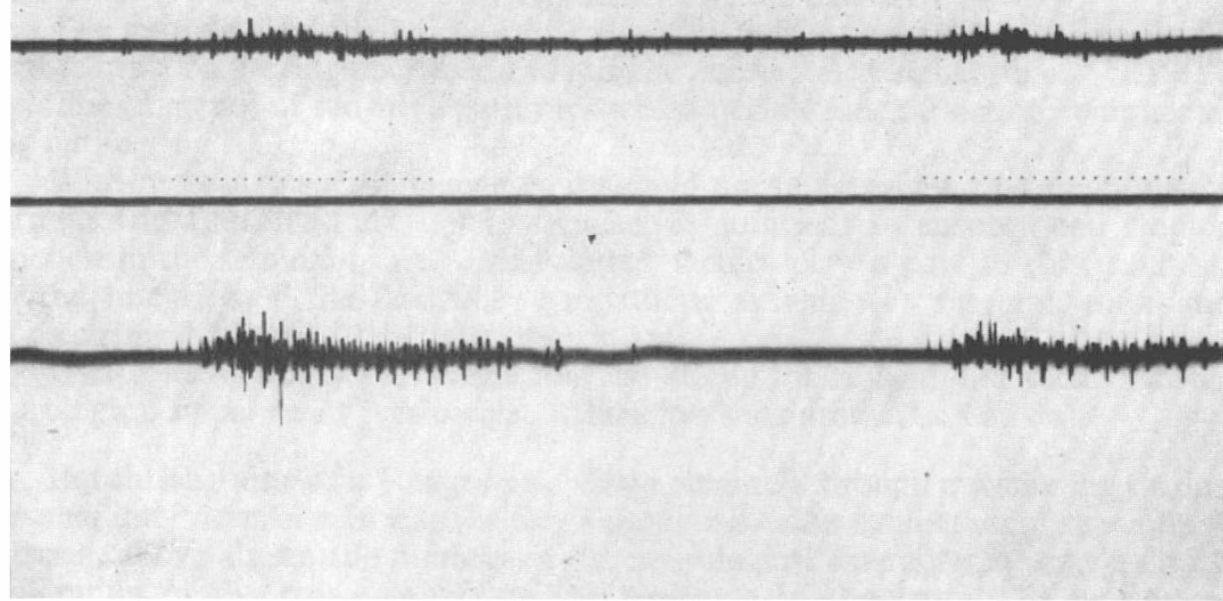

FIG. I5

The top tracing shows activity taken from the s.a., and the lower tracing activity taken from the s.v. by direct stimulation of the sacral roots with electrodes which were inserted through the Hiatus sacralis (frequency of stimulation $22 / \mathrm{sec}$.). 
activity is also diminished, or, in cases of a complete transverse lesion of the spinal cord, it is completely extinguished. However, the only activity which is diminished or extinguished is that which is induced by voluntary innervation (fig. I0).

On the other hand, reflex activity remains unchanged as in the case of normal controls or sometimes even it is intensified (fig. II, coughs), (fig. I2, rhythmic pressure on the abdomen), (fig. I3, bulbocavernosus reflex).

Thus, in the case of upper motor neuron lesions, we find the following: The greater the extent of the lesion, the weaker the voluntarily induced electrical activity, while, on the other hand, reflex activity remains unchanged (fig. I4). This is of great importance since such activity induced by coughing or pressure on the abdomen could mimic an unimpaired impulse transmission.

By inserting a stimulation electrode through the Hiatus sacralis into the region of the lower sacral roots, we can stimulate these roots. This is of importance in cases of lesions of the peripheral nerves in order to determine whether or not the roots can be stimulated. In this way, we can judge the extent of the lesion by measuring the latency and the intensity of the response for the stimulus (fig. 15).

In cases of lesions above the sacral spinal cord, that is, when the sacral motor centres remain intact, the stimulation responses remain normal.

\section{SUMMARY}

By means of an EMG tracing from the striated sphincters in cases of neurogenic bladder and rectal disturbances, the following can be determined:

(I) if a disturbance of innervation is present or not

(2) if this lesion affects the central or peripheral motor neuron.

(3) the extent of the lesion. 